\title{
Transmission and expression of the parasitic paternal sex ratio (PSR) chromosome
}

\author{
LEO W. BEUKEBOOM* \& JOHN H. WERREN \\ Department of Biology, University of Rochester, Rochester, NY 14627, U.S.A.
}

\begin{abstract}
B-chromosomes are often considered genomic parasites. They are extra to the normal chromosomal complement, are unnecessary for survival of an individual, and are often inherited at higher than Mendelian rates. Paternal Sex Ratio (PSR) is an extreme example of a parasitic B-chromosome in the wasp Nasonia vitripennis. It is transmitted via sperm but then destroys the other paternal chromosomes in the early fertilized egg. PSR disrupts the normal haplodiploid sex determination system of this wasp by converting diploid (female) eggs into haploid eggs that develop into PSRbearing males. Transmission and expression of PSR was measured in single pair crosses between carrier males and standard females. Presence of the chromosome was detected by probing offspring with PSR-specific repetitive DNA. Most (equal to or more than 90 per cent).PSR males produced all-male offspring. Overall transmission rates of PSR to fertilized eggs varied beween 0.94 and 1.0. Some males (up to 10 per cent) produced daughters at varying frequencies. Of 226 daughters tested, only one carried PSR (and this may have been a laboratory error) indicating that daughters result from failure of transmission rather than loss of expression. Transmission of PSR to males in families that included female offspring varied from 0 to 94 per cent. Incomplete transmission is most likely the result of loss of PSR in some spermatogonial cell lineages and indicates some mitotic instability. Implications of the results to the aetiology and population genetics of PSR are discussed.
\end{abstract}

Keywords: B-chromosome, mitotic stability, Nasonia, Paternal Sex Ratio, population genetics, selfish DNA.

\section{Introduction}

B-chromosomes are extra, non-vital chromosomes which differ in number between individuals of the same species. They are unnecessary for survival and reproduction and are generally regarded as genetically inert based on their small size and heterochromatic state. B-chromosomes are estimated to occur in 10-15 per cent of all eukaryotic species (Jones \& Rees, 1982; Harvey, 1989). Most B-chromosomes gain a higher than Mendelian transmission through preferential segregation to functional gametes at meiosis or directed non-disjunction during mitosis (Jones, 1985). Although Östergren (1945) originally suggested their parasitic nature, B-chromosomes have long been regarded as beneficial to the organisms carrying them (Darlington, 1956; Rees \& Ayonoadu, 1973; White, 1973; but see Melander, 1950; Muntzing, 1961;

${ }^{*}$ Correspondence: Arbeitsgruppe Michiels, Max-Planck-Institut für Verhaltensphysiologie, D-8130 Seewiesen (Post Starnberg), Germany.
Kimura \& Kayano, 1961; Nur, 1962, 1966, 1977). Only recently have they received wider recognition as genomic parasites (i.e. Jones, 1985; Werren et al., 1988; Bell \& Burt, 1990; Shaw \& Hewitt, 1990).

Paternal Sex Ratio (PSR) is an extreme example of a parasitic B-chromosome (Werren et al., 1987; Nur et al., 1988; Werren, 1991). It occurs in some natural populations of the parasitoid wasp Nasonia vitripennis. Males who carry PSR transmit it via sperm to fertilized eggs. PSR then causes improper condensation and subsequent loss of remaining paternal chromosomes (Werren et al., 1987; Nur et al., 1988). Nasonia has haplodiploid sex determination. Normally, fertilized (diploid) eggs develop into females and unfertilized (haploid) eggs develop into males. The effect of PSR is to convert diploid eggs, which would normally develop into females, into haploid eggs that develop into PSRbearing males. Although the other paternal chromosomes are destroyed, PSR is itself transmitted and will eliminate the new set of chromosomes, derived from the mother, in the next generation. Because PSR destroys all chromosomes with which it is associated 
every generation, it is the most selfish genetic element so far described (Werren et al., 1988; Godfray \& Harvey, 1989).

The PSR chromosome differs from standard B-chromosomes in several ways. First, it is the only known B-chromosome that destroys the genome of its carrier. Second, it occurs only in males, whereas other B-chromosomes are usually transmitted through both sexes. Third, because PSR results in the production of males, it has a clear phenotype by which it can be detected (i.e. Werren \& van den Assem, 1986, Beukeboom \& Werren, 1992a). PSR can also be detected by using PSR-specific repetitive DNA probes (i.e. Nur et al., 1988; Eickbush et al., 1992; Beukeboom \& Werren, 1992). PSR can also be detected by using PSR-specific repetitive DNA probes (i.e. Nur et al., 1988; Eickbush et al., 1992; Beukeboom \& Werren, 1992). Expression of PSR independent measures of PSR transmission and expression.

From a parasitic point of view, the frequency of $\mathrm{B}$-chromosomes in populations is considered a balance between their transmission drive and their negative effects on host fitness (Kimura \& Kayano, 1961; Nur, 1969, 1977; Hewitt, 1973; Shaw, 1984). Therefore, detailed information on transmission rates also increases our understanding of how PSR is maintained in natural populations. The present study was designed to measure expression and transmission rates of PSR. The results are informative about the mode of PSR action and inheritance.

\section{Methods}

\section{Strains}

All experiments were carried out with the parasitoid wasp Nasonia vitripennis. Our standard wild-type strain is Leiden Labll (subsequently referred to as 1.abII or Standard). In addition to the LabII strain, a high fertilization strain (MI, Macomb, Illinois; Saul et al., 1965) was used. This strain carries the Maternal Sex Ratio (MSR) distorter, which is a maternally-transmitted cytoplasmic element that causes females to fertilize 90-100 per cent of their eggs (Skinner, 1982). The causative agent of MSR is unknown. An advantage of using a high fertilization strain for PSR maintenance is high transmission of PSR to offspring through fertilized eggs. The PSR chromosome was maintained in a LabII background, designated as PSR(LabII). The standard PSR chromosome from previous studies was used (Werren \& van den Assem, 1986; Beukeboom \& Werren, 1992).

\section{Standard culturing methods}

Wasps were maintained at $25^{\circ} \mathrm{C}$ on Sarcophaga bullata hosts. Generation time was approximately 14 days. Virgin females and males were collected and isolated in the pupal stage by opening Sarcophaga hosts 11-13 days after egg laying. Adult wasps were usually 1 day old when used in experiments. Because females are unable to use sperm during the first hours after mating (van den Assem \& Feuth-de Bruin, 1977), mated females were not given hosts for the first $24 \mathrm{~h}$ following mating.

\section{PSR screening methods}

A molecular assay (dot-blot assay) based on DNA hybridization techniques was used to screen for PSR (described in detail in Beukeboom \& Werren, 1992). Wasps were ground in homogenization buffer, the solution denatured and neutralized, and $1 \mu \mathrm{l}$ dotted onto nitrocellulose filters. Filters were hybridized with a ${ }^{32} \mathrm{P}-$ labelled probe of PSR-specific repetitive DNA. After exposing the filters to autoradiographic film, PSR-carrying individuals could easily be identified, because normal males give no signal. It should be noted that this is a retrospective procedure; it can only identify PSR males after they have been used in experiments and sacrificed.

\section{Measuring PSR transmission rates}

Two experiments were performed to measure transmission and expression of PSR. PSR can only be transmitted to fertilized eggs. Females typically produce some unfertilized eggs (0-10 per cent in the MI strain; 0-25 per cent in the LabII strain under the experimental conditions). The first experiment compared proportion of PSR males among progeny in PSR matings with proportion of fertilized eggs (daughters) in control crosses. Sometimes, PSR males produce daughters (Werren \& van den Assem, 1986). The second experiment measured the frequency of daughter production by PSR males and determined whether PSR was transmitted to sons and daughters in these progenies.

Experiment I. There were $22 \mathrm{PSR}(\mathrm{L}$ abII) and 40 Standard (LabII) males individually mated with MI virgin females. Presence of PSR among male parents was subsequently confirmed in a dot-blot assay. Females were provided with hosts for oviposition twenty four hours later. Progeny sex ratios were scored and males from PSR progenies were individually dotblotted to determine PSR frequency. 
Experiment II. In all, 418 males were taken from PSR (LabII) all-male families and individually mated with LabIl virgin females. The next day, females were provided with a host for oviposition and the male was removed and probed to determine whether he carried the PSR chromosome. The presence of PSR among offspring was also scored by dot-blotting. Depending on the sex ratio of the progeny, either a subset or all of the sons and daughters in each progeny were probed. Control crosses (LabII males $\times$ LabII females, $n=40$ ) were performed to estimate the proportion of fertilized eggs produced by LabII females, This experiment determines rates of daughter production by PSR males, and whether these daughters carry the PSR chromosome.

\section{Results}

Rates of transmission and expression of PSR were investigated in two experiments.

\section{Experiment I}

All 22 PSR(LabII) males (confirmed by molecular probing) individually mated to MI females, produced all-male families in this experiment. This is the typical phenotype of PSR. All progeny from each of these families were individually probed to determine presence of PSR. Results are shown in Table 1. As can be seen, PSR families were around 14 per cent larger than control families, although this difference was nonsignificant. The number of wild-type (non-PSR) males was the same in control and PSR families. These are presumably derived from unfertilized (haploid) eggs. Similarly, the proportion of PSR among male progeny in PSR crosses $(0.984 \pm 0.031$ s.d.) did not differ significantly from the proportion of daughters in control crosses (i.e. fertilized eggs). This indicates that transmission of PSR to fertilized eggs was near 100 per cent.

The ratio, proportion PSR sons/proportion control daughters, gives an estimate of PSR transmission to fertilized eggs. The estimate is 1.015 with 95 per cent confidence limits of 1.005 to 1.025 , using the Delta method for estimating the variance of ratios (Crow \& Kimura, 1970). In biological terms, the transmisson rate is 1.0 , or 100 per cent to fertilized eggs. It is interesting that the confidence limits fall above 1.0, which is biologically impossible. We believe this occurs because our estimate of fertilization rate in controls is based on the proportion of daughters among adult progeny. If females have a higher egg to adult mortality than do males, the fertilization rates would actually be slightly higher than estimated. Comparing the number of control females to PSR males in Table 1 indicates that female progeny have around 86 per cent the survival of PSR males. Other experiments (Beukeboom, 1993) have found a significant increase of 10-20 per cent in PSR family sizes relative to controls, again suggesting slightly higher mortality rates for female progeny. An unlikely alternative is that PSRmated females actually lay more eggs.

\section{Experiment II.}

Several studies (Werren \& van den Assem, 1986; Skinner, 1987) have found a fraction of PSR males to

Table 1 Mean number of non-PSR males, females and PSR males in progenies from non-PSR (control) mated females and PSR-mated females in Experiment I. Because of haplodiploidy, unfertilized (haploid) eggs normally develop into (nonPSR) males and fertilized (diploid) eggs into females (control crosses). PSR is transmitted via sperm of carrier males and haplodizes fertilized eggs by destroying the paternal set of chromosomes. As a consequence, PSR fertilized eggs develop into PSR males instead of females. The proportion of fertilized eggs in control is the proportion daughters, whereas in PSR crosses it is the proportion PSR sons

\begin{tabular}{lccccc}
\hline & Control & PSR & Mann-Whitney $U$-test \\
\hline Families & $n=40$ & $n=22$ & & & \\
Non-PSR males & $1.13 \pm 1.79$ & $0.64 \pm 1.18$ & $z=-1.245$ & $P=0.213$ \\
Females & $33.73 \pm 8.08$ & - & & & \\
PSR males & - & $39.14 \pm 11.85$ & $z=1.554$ & $P=0.120$ \\
Total & $34.85 \pm 8.16$ & $39.77 \pm 11.84$ & $z=1.429$ & $P=0.153$ \\
Proportion fertilized & $0.968 \pm 0.047$ & $0.984 \pm 0.031$ & $z=1.317$ & $P=0.188$ \\
$\quad$ & & & & & \\
\hline
\end{tabular}

Values are \pm s.d. Differences are tested with the Mann-Whitney $U$-test. None of the differences is significant at the 5 per cent level. 
produce some female progeny, indicating partial expression of PSR. To quantify daughter production and PSR transmission among known PSR males, the following experiment was conducted. From PSR(LabII) all-male families 418 males were individually mated to virgin LabII females and subsequently probed for PSR. Of these, 379 males probed positive for PSR. The remaining 39 (9.3 per cent) were presumably derived from unfertilized eggs, which cannot inherit PSR (Werren \& van den Assem, 1986).

Table 2 shows the sex ratios among progeny of the PSR-positive males. As can be seen, most (90.2 per cent) produced all-male families. However, around 10 per cent of PSR males produced some daughters among their progeny. There was 5 per cent of PSR males that actually produced female-biased (more than 75 per cent female) sex ratios typical of wild-type LabII males (control LabII $\times$ LabII matings, $0.864 \pm 0.073$ s.d., $n=40$ families). Another 5 per cent produced intermediate sex ratios (1-75 per cent females). A sample of male and female progeny from each sex ratio category was probed to determine rates of transmission of PSR to both sexes.

PSR was not transmitted to daughters $(n=226$ tested), except for one case of weak hybridization in the 11-50 per cent female group. This case is most likely the result of a spontaneous mutation to nonfunctionality of the PSR chromosome. Non-functional PSR chromosomes, resulting in transmission to females, have been created by mutagenesis and are transmitted to females (Beukeboom \& Werren, 1993). Such chromosomes are often mitotically unstable, resulting in individuals that are somatic mosaics for the chromosome and that show weak hybridization on dotblots (Beukeboom et al., 1992). Therefore, we conclude that transmission of PSR without expression (i.e. destruction of the paternal chromosomes causing conversion of fertilized eggs to male) is a rare event.

Transmission of PSR to male progeny is negatively correlated with the sex ratio (proportion females; Spearman rank correlation, $r_{\mathrm{s}}=-1.00, P=0.01, n=$ five categories). In all-male families, the proportion PSR sons was equal to the fertilization proportion in controls (0.879 vs. 0.864$)$. Among PSR males that produced progenies with proportion daughters ranging from 1 to 75 per cent ( 4.8 per cent, $n=18$ ), PSR was transmitted to sons at rates ranging from 94 to 17 per cent. Fertilization proportions in those progenies (i.e. proportion daughters + PSR sons) varied from 0.840 to 0.876 (vs. 0.864 in control crosses). PSR males that produced 'normal' female-biased sex ratios (equal to or more than 75 per cent females) (5.0 per cent, $n=19$ )

Table 2 Sex ratıos of progenies from PSR crosses in Experiment 1I. PSR males produce either all-male, various (1-75 per cent female) or 'normal' female-biased (equal to or more than 75 per cent female) sex ratio progenies. Transmission rates of PSR to sons and daughters are given for each category and for all categories pooled. PSR transmission is negatively correlated with the frequency of female progeny. In some cases, only a subset of progeny was individually screened for presence of PSR

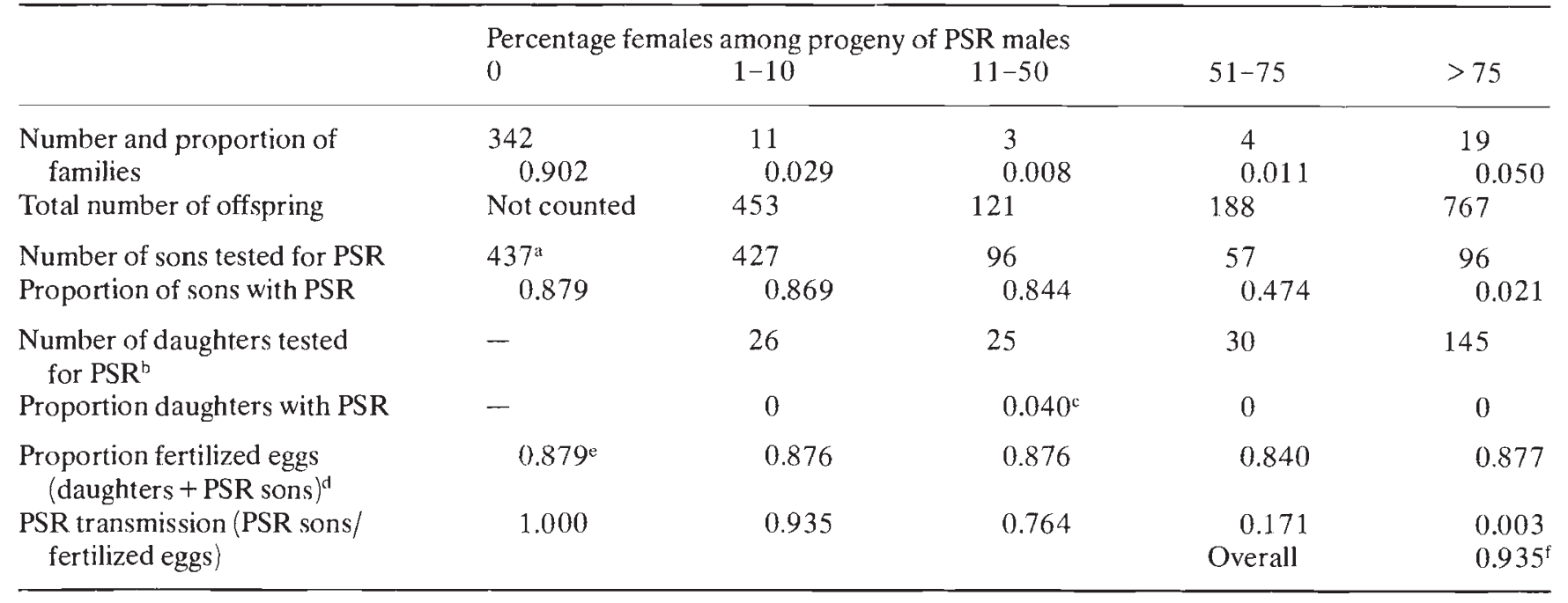

${ }^{a}$ Subset of the total number of males in these families.

bubset of the total number of females in these families.

' Probably a spontaneous mutation to non-functionality of PSR.

${ }^{d}$ Fertilization in control crosses was $0.864 \pm 0.073$ s.d.

${ }^{\text {e }}$ Determined from proportion PSR among tested males.

${ }^{\mathrm{f}}$ Proportion PSR sons divided by proportion fertilized eggs, summed for all sex ratio categories. 
did not transmit PSR to any of their sons, except for one male which produced five sons of which two probed positive for PSR. Overall PSR transmission among these progenies was 0.3 per cent. Proportion fertilized eggs was 0.877 (females plus PSR males), which is similar to controls. Overall PSR transmission to fertilized eggs, estimated by dividing the proportion PSR males by the proportion fertilized eggs (PSR males + daughters) and then summing over all sex ratio categories, was 0.935 (Table 2). These data indicate that all fertilized eggs that do not develop into PSR males develop into daughters.

Interestingly, PSR fathers that produced female progeny were much more likely to probe weakly for the chromosome than were those producing all-male families. For example, 13 of 19 (68 per cent) PSR males that produced sex ratios of more than 75 per cent female probed weakly for PSR, vs. 1 of 18 (6 per cent) producing 1-75 per cent females and 12 of 342 (4 per cent) producing all-male families. Males probing weakly for PSR are likely to be somatic and germline mosaics, based on studies of deletion chromosomes (Beukeboom et al., 1992).

\section{Discussion}

PSR is an exceptional B-chromosome because it gains its transmission drive by eliminating the paternal chromosomes in the fertilized egg each generation. This study found individual variation in transmission rates of PSR to offspring. Occasionally, some males that probed positive for PSR failed to transmit the chromosome to their progeny. Overall transmission rates in this study were 1.0 in one experiment and 0.935 in another experiment. These values are similar to previous reported rates of $0.9-1.0$ (Werren \& van den Assem, 1986), which were obtained before development of the molecular screening method for PSR. In that study, individuals producing more than 90 per cent male families were scored as PSR positive. This phenotypic assay is not completely accurate for two reasons. First, the current study clearly shows that some (about 7 per cent) PSR males produce progenies with fewer than 90 per cent males. Second, owing to haplodiploidy, all-male families can also be produced by virgin females.

Our findings indicate that whenever PSR is transmitted, it is expressed. Loss of expression would result in female progeny that carry the chromosome. With one exception out of 226 females tested, this did not occur. The one female that probed positive could have resulted from inheritance of a spontaneously mutated PSR chromosome. Non-functional PSR chromosomes can be generated by mutagenesis and are transmitted to female progeny (Beukeboom \& Werren, 1992b). Consistent with this interpretation, the chromosome appeared to be mitotically unstable based on weak hybridization, which is a characteristic of non-functional PSR deletion chromosomes (Beukeboom et al., 1992). However, this female could also represent a laboratory misidentification.

PSR was not transmitted to 100 per cent of fertilized eggs in these experiments. Around 10 per cent of males probing positive for PSR actually produced some female progeny, who did not inherit PSR. These males also showed reduced rates of PSR transmission. Daughter production among PSR males is probably a result of mitotic loss of the PSR chromosome during development (Beukeboom et al., 1992). This results in mosaic individuals, in which the number of cells carrying PSR depends on the developmental stage at which loss occurs. Consistent with this view is the observation that PSR males producing female-biased sex ratios often probed weakly for PSR. Transmission and expression of PSR in mosaic individuals will depend on whether PSR is present in their germ-line tissue. Mosaicism in the testes would lead to production of PSR-minus sperm, which apparently function normally and result in (PSR-minus) female progeny. Based on this interpretation, the PSR males which produced 1-75 per cent female progeny were germ-line mosaics for PSR. The males that probed positive for PSR but produced normal sex ratios (more than 75 per cent female) had no PSR transmission to progeny (except for one male who produced two PSR sons). They were apparently somatic mosaics, with PSR completely absent in germ-line tissue. This would result, for instance, if the PSR chromosome were lost in some cell lineages very early in development.

The data indicate that whenever the PSR chromosome is expressed, it is also transmitted. There are two possible ways in which males that originated from PSR action could have been scored as PSR negative. If PSR action is caused by imprinting of the paternal chromosomes during spermatogenesis, then loss of the PSR chromosome after imprinting could result in expression without transmission. If PSR were lost very early during development of the PSR fertilized egg, then the PSR chromosome may have been present in too few cells to be detected in the dot-blot assay. Expression without transmission would result in an increase in the proportion of PSR-minus males relative to control matings. This did not occur. In addition, PSR males that incompletely transmitted the chromosome produced daughters at frequencies expected if all fertilized eggs resulted either in female or PSR male progeny.

B-chromosomes are widespread, being found in over 1000 animal and plant species (Jones \& Rees, 
1982). PSR differs from all other B-chromosomes in its extreme phenotype. Most B's have relatively small effects on host fitness (at least at low copy number) and gain a transmission advantage by non-random segregation during meiosis or mitosis. PSR gains a transmission advantage by totally eliminating the paternally-derived genome, except itself. Such an extreme phenotype can only evolve under haplodiploidy, where males develop from haploid eggs.

Finally, the results are relevant to population dynamics of PSR. Because PSR is only transmitted to fertilized eggs, greater than 50 per cent eggs must be fertilized for PSR to exist in any population (Werren, 1987; Skinner, 1987; Werren \& Beukeboom, 1993). Nasonia populations are divided into local mating groups or demes in nature (Skinner, 1983; Werren, 1983). Beukeboom \& Werren (1992) showed that highly subdivided population structure has opposing effects on PSR. First, owing to local mate competition (Hamilton, 1967; Werren, 1983) female Nasonia fertilize more eggs in smaller foundress groups. This increases PSR frequency. However, when PSR becomes abundant in a deme the number of mates per male decreases and the chance of local extinction of demes solely founded by PSR-mated females increases. These negative effects on PSR are most strongly expressed in small demes. One of the key assumptions in our population models is that PSR transmission to fertilized eggs is at or near 1.0. This study confirms transmission rates of PSR to be 0.94-1.0, consistent with previous phenotypic estimates.

Differences in transmission rates of B-chromosomes between individuals have been observed in several species (i.e. Hewitt, 1973; Parker et al., 1982; Matthews \& Jones, 1983; Shaw \& Hewitt, 1985; Shaw et al., 1985; Puertas et al., 1990; Viseras, 1990; Romera et al., 1991). Nur \& Brett $(1985,1987,1988)$ have demonstrated that this results from the existence of genetic factors affecting the condensation of the B-chromosome in the mealy bug Pseudococcus. Attempts at selecting lines for low and high transmission of PSR have been unsuccessful (L. W. Beukeboom, unpublished data). Factors that control mitotic stability of wild-type PSR chromosomes are currently not known. However, reduced transmission rates or stability of PSR could be important in natural populations, where selection for autosomal modifiers (repressors) of PSR transmission and expression may occur.

\section{Acknowledgements}

We thank Kent Reed for valuable comments on the manuscript and Wyatt Anderson for statistical advice.
This research was supported by NSF grant BSR8796222. L.W.B. acknowledges financial support from a Fulbright fellowship, the 'Dobberke Stichting' and the 'Donders Fonds'.

\section{References}

ASSEM, J. VAN DEN AND FEUTH-DE BRUIJN, E. 1977. Second matings and their effect on the sex ratio of the offspring in Nasonia vitripennis (Hymenoptera: Pteromalidae). Entomol. Exp. App., 21, 23-28.

BeUkeboom, L. w. 1993. Phenotypic fitness effects of the parasitic Paternal Sex Ratio (PSR) chromosome. Evol. Ecol., in press.

BEUKeboOM, L. M. AND WERren, J. H. 1992a. Population genetics of a parasitic chromosome: experimental analysis of PSR in subdivided populations. Evolution, 46,1257-1268.

BEuKeboom, L. W. AND WERREN, J. H. 1993. Deletion analysis of a parasitic B-chromosome - Paternal Sex Ratio (PSR). Genetics, in press.

BEUKEBOOM, L. W., REED, K. M. AND WERREN, J. H. 1992. Effects of deletions on mitotic stability of the Paternal Sex Ratio (PSR) chromosome from Nasonia. Chromosoma, 102, 20-26.

BELL, G. AND BURT, A. 1990. B-chromosomes: germ-line parasites which induce changes in host recombination. Parasitology, 100, S19-S26.

Crow, J. F. ANd Kimura, M. 1970. An Introduction to Population Genetics. Alpha Editions, Burgess Publishing Company, Minneapolis.

DARLington, C. D. 1956. Chromosome Botany. Allen and Unwin Ltd, London.

EICKBUSH, D. G., EICKBUSH, T. H. AND WERREN, J. H. 1992. Molecular characterization of repetitive DNA sequences from a B-chromosome. Chromosoma, 101, 575-583.

GODFRAY, H. C. J. AND HARVEY, P. H. 1989. Seed of destruction. Nature, 337, 210-211.

HAMILTON, W. D. 1967. Extraordinary sex ratios. Science, 155, 477-488.

HARVEY, P. H. 1989. Parasitological teeth for evolutionary problems. Nature, 342, 230.

HEWITT, G. M. 1973. Variable transmission rate of a B-chromosome in Myrmeleotettix maculatus (Thumb.) (Acrididae: Orthoptera). Chromosoma, 40, 83-106.

JONES, R. N. 1985. Are B-chromosomes selfish? In: Cavalier Smith, T. (ed.) The Evolution of Genome Size, John Wiley, Chichester, pp. 397-425.

JONES, R. N. AND REES, H. 1982. B. Chromosomes. Academic Press, London.

KIMURA, M. AND KAYANO, H. 1961. The maintenance of supernumerary chromosomes in wild populations of Lilium callosum by preferential segregation. Genetics, 46, 1699-1712.

MATTHEWS, R. B. AND JONES, R. N. 1983. Dynamics of the B chromosome polymorphism in rye. II. Estimates of parameters. Heredity, 50, 119-137.

MELANDER, Y. 1950. Accessory chromosomes in animals, especially in Polycleis tenuis. Hereditas, 36, 19-38. 
MunTzing, A. 1961. Genetical Research. LTs Forlag, Stockholm.

NUR, U. 1962. Population studies of supernumerary chromosomes in a mealybug population. Genetics, 47, 16791690.

NUR, U. 1966. Harmful supernumerary chromosomes in a mealybug population. Genetics, 54, 1225-1238.

NUR, U. 1969. Harmful B-chromosomes in a mealybug: additional evidence. Chromosoma, 28, 380-397.

NUR, U. 1977. Maintenance of a 'parasitic' B chromosome in the grasshopper Melanoplus femur-rubrum. Genetics, 87, 499-512.

NUR, U. AND BRETT, B. L. H. 1985. Genotypes suppressing meiotic drive of a B-chromosome in the mealybug, Pseudococcus affinis. Genetics, 110, 73-92.

NUR, U. AND BRETT, B. L. H. 1987. Control of meiotic drive of a B-chromosome in the mealybug, Pseudococcus affinis (obscurus). Genetics, 115, 499-510.

NUR, U. AND BRETT, B. L. H. 1988. Genotypes affecting the condensation and transmission of heterochromatic Bchromosomes in the mealybug, Pseudococcus affinis. Chromosoma, 96, 205-212.

NUR, U., WERREN, J. H., EICKBUSH, D., BURKE, W. AND EICKBUSH, T. 1988. A 'selfish' B chromosome that enhances its transmission by eliminating the paternal chromosomes. Science, 240, 512-514.

Ostergren, G. 1945. Parasitic nature of extra fragment chromosomes. Botaniska Notiser, 2, 157-163.

PARKER, J. S., TAYLOR, S. AND AINSWORTH, C. C. 1982. The B-chromosome system of Hypochoeris maculata. III. Variation in B-chromosome transmission rates. Chromosoma, 85, 299-310.

PUERTAS, M. J., JMENEZ, M. M., ROMERA, F., VEGA, J. M. AND DIEZ, M. 1990. Maternal imprinting effect on B-chromosome transmission in rye. Heredity, 64, 197-204.

REES, H. AND AYONOADU, U. 1973. B-chromosome selection in rye. Theoret. Appl. Genet., 43, 162-166.

ROMERA, F., JIMÉNEZ, M. M. AND PUERTAS, M. J. 1991. Genetic control of the rate of transmission of rye $\mathrm{B}$ chromosomes. I. Effects in 2B $\times 0 \mathrm{~B}$ crosses. Heredity, 66, 61-65.

SAUL, G. B. 2nd, WHITING, P. W., SAUL, s. W. AND HEIDNER, C. A. 1965. Wild-type and mutant stocks of Mormoniella. Genetics, 52, 1317-1327.

SHAW, M. w. 1984. The population genetics of the B chromosome of Myrmeleotettix maculatus (Thunb.) (Orthoptera: Acrididae). Biol. J. Linn. Soc., 23, 77-100.
SHAW, M. W. AND HEWITT, G. M. 1985. The genetic control of meiotic drive acting on the B-chromosomes of Myrmeleotettix maculatus (Orthoptera: Acrididae). Heredity, 54, 187-194.

SHAW, M. W. AND HEWITT, G. M. 1990. B chromosomes, selfish DNA and theoretical models: where next? In: Futuyma, D. and Antonovics, J. (eds) Oxford Surveys in Evolutionary Biology, vol. 7, Oxford University Press, pp. 197-223.

Shaw, M. W., HewitT, G. M. AND ANDERSON, D. A. 1985. Polymorphism in the rates of meiotic drive acting on the B-chromosome of Myrmeleotettix maculatus. Heredity, 55, 61-68.

SKINNER, S. W. 1982. Maternally inherited sex ratio in the parasitoid wasp, Nasonia (= Mormoniella) vitripennis. Science, 215, 1133-1134.

SKINNER, S. w. 1983. Extrachromosomal sex ratio factors in the parasitoid wasp Nasonia (= Mormoniella) vitripennis. Ph.D Dissertation, University of Utah, Salt Lake City, U.S.A.

SKINNER, S. w. 1987. Paternal transmission of an extrachromosomal factor in a wasp: evolutionary implications. Heredity, 59, 47-53.

VISERAS, E., PARDO, M. C. AND CAMACHO, J. P. M. 1990. Analysis of B-chromosome transmission through females of Locusta migratoria. Bull. Genet. Soc. Can., 21 s, 28.

WERREN, J. H. 1983. Sex ratio evolution under local mate competition in a parasitic wasp. Evolution, 37, 116-124.

WERREN, J. H. 1987. The coevolution of autosomal and cytoplasmic sex ratio factors. J. Theoret. Biol., 124, 317-334.

WERren, J. H. 1991. The Paternal-Sex-Ratio chromosome of Nasonia. Amer. Nat., 137, 392-402.

WERREN, J. H. AND VAN DEN ASSEM, J. 1986. Experimental analysis of a paternally inherited extrachromosomal factor. Genetics, 114, 217-233.

WERREN, J. H., NUR, U. AND EICKBUSH, D. 1987. An extrachromosomal factor causing loss of paternal chromosomes. Nature, 327, 75-76.

WERREN, J. H. AND BEUKeboom, L. W. 1993. Population genetics of a parasitic chromosome. Theoretical analysis of PSR in subdivided populations. Amer. Nat., in press.

WERREN, J. H., NUR, U. AND WU, C.-1. 1988. Selfish genetic elements. Trends Ecol. Evol., 3, 297-302.

whITE, M. J. D. 1973. Animal Cytology and Evolution, 3rd edn. Cambridge University Press, Cambridge.

Whiting, A. R. 1967. The biology of the parasitic wasp, Mormoniella vitripennis. Q. Rev. Biol., 43, 333-406. 